\title{
Waveform considerations in space-variant optical processors
}

\author{
David Casasent and Demetri Psaltis \\ Department of Electrical Engineering, Carnegie-Mellon University, Pittsburgh, Pennsylvania 15213
}

Received August 24, 1978

\begin{abstract}
The use of coded waveforms in space-variant optical signal processors using coordinate transformations is considered. It is shown that nonlinear transmitted coded signals must be used with such a processor and that this results in novel waveform design and system approaches for radar and communications.
\end{abstract}

Space-variant optical processors are the subject of considerable research. ${ }^{1}$ One of the most promising methods by which such systems can be realized is the application of coordinate transformations to the input data. ${ }^{2,3}$ The extension of this technique to produce optical Mellin transforms ${ }^{4}$ and distortion-invariant pattern-recognition systems has been reported over the past three years. ${ }^{5-7}$ Of particular interest in this Letter is the use of the Mellin transform in Doppler-invariant signal processors, as we described earlier.8,9 However, because the Mellin transform is a space-variant operation, a time-sequential implementation of the required coordinate transformation would be required unless a fully parallel, real-time, coordinate transformation system can be developed. With such a properly configured system, a single-channel 1-D optical processor can be used to realize the necessary range/Doppler processing.

At least two techniques have been reported by which the required parallel coordinate transformation can be realized. One scheme that is appropriate for 2-D inputs utilizes computer-generated holograms ${ }^{10-12}$ but suffers from input space-bandwidth product limitations. A second method ${ }^{13,14}$ is of use with 1-D input functions and 1-D distortions only and results in large light losses. However, with refinements in these methods, our single-channel 1-D, real-time Mellin-transform Dopplerinvariant-processor concept ${ }^{8,9}$ seems more realizable.

Hence, in this Letter, we extend our earlier work ${ }^{8,9}$ to the case of coded waveforms, as are conventionally used in radar and communications. We now describe in more detail the waveform properties and operation of such space-variant optical processors with complex coded waveforms.

Following a review of Mellin-transform-based Doppler-invariant signal processing we show that previously described space-variant signal processors $8,9,15$ cannot operate on conventional coded waveforms without a severe loss in system performance. Rather, proper operation of such processors requires transmission codes that are nonlinear. Such waveforms are then shown to result in a novel spread-spectrum coding that promises to provide new research avenues for waveform design in spread-spectrum, radar, and communications systems. As we have long advocated, optical signal processors should not be limited by the conventional waveform and system-design constraints introduced by existing processing technologies. The case at hand serves to demonstrate this point vividly. In future papers, we will describe the ambiguity functions, system-design parameters, and the advantageous noise, jammer, and interference-rejection features of such systems using nonlinear waveforms and space-variant optical processors.

In the general formulation of space-variant pattern recognition, we represent the undistorted input signal (in 1-D for simplicity) by $f(x)$ and a distorted version of it by

$$
f^{\prime}(x)=f[g(x, a)],
$$

where $g(x, a)$ is the distorting function and $a$ an unknown distortion parameter. To correlate $f(x)$ and $f^{\prime}(x)$ invariant to the distortion $g(x, a)$, we apply the coordinate transformation ${ }^{7}$

$$
t=h^{-1}(x)=\frac{\mathrm{d} t_{0}}{\mathrm{~d} a} \int_{-\infty}^{x} \frac{\partial g / \partial x}{\partial g / \partial a} \mathrm{~d} x
$$

to $f(x)$ and $f^{\prime}(x)$, where $t_{0}$ is a function of $a$ alone. This transformation produces two new functions, $f_{1}(t)$ and $f_{1}(t)$, which when correlated yield ${ }^{4-9}$

$$
R(\hat{t})=f_{1}(t) \circledast f_{1}^{\prime}(t)=f_{1}(t) \circledast f_{1}(t) * \delta\left(\hat{t}-t_{0}\right),
$$

where $\circledast$ denotes correlation, * represents convolution, and $\hat{t}$ is the correlation plane variable. The unknown distortion parameter $a$ can be found from the location $\left(\hat{t}=t_{0}\right)$ of the output correlation peak.

When the input distortion is $g(x, a)=a x, f^{\prime}(x)=f(a x)$ is a Doppler-shifted version of $f(x)$. Solving Eq. (2), we find that $t=\ln x$ and $t_{0}=\ln a$. Thus the resultant system of Fig. 1 with $f_{1}{ }^{\prime}(t)$ recorded at $P_{0}$ and $F_{1}{ }^{*}(u)=$ $\mathscr{F}\left[f_{1}(t)\right]$ recorded at $P_{1}$ becomes a space-variant Doppler-invariant 1-D signal processor in which $\hat{t}$ corre-

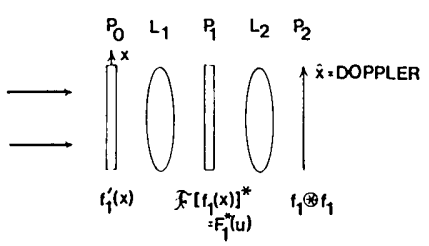

Fig. 1. Schematic diagram of a space-variant, Doppler-invariant optical signal processor. 


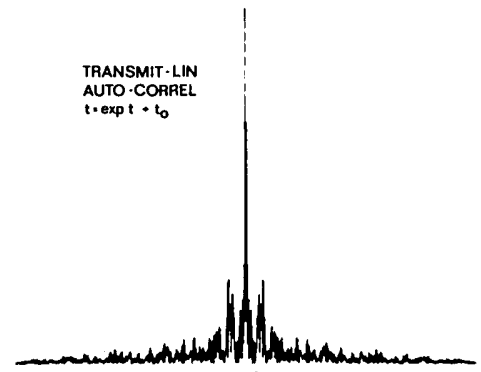

(a)

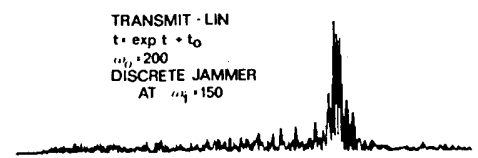

(b)

Fig. 2. Cross-sectional scan of the optically produced correlation of a nonlinear coordinate-transformed Barker-coded waveform: (a) autocorrelation, (b) jammer.

sponds to the Doppler channel. With a time-varying input function at $P_{0}$, the time of occurrence of the $P_{2}$ output peak denotes the target's range, whereas the spatial position of the peak denotes its Doppler.

We now concern ourselves with the performance of the system of Fig. 1 when $f(t)$ is a coded waveform. For the case of binary phase-modulated waveforms with constant amplitude modulation, we represent the real form of $f(t)$ by

$$
f(t)=\cos \left[\omega_{0} t+\phi(t)\right]=a(t) \cos \left(\omega_{0} t\right)
$$

where $a(t)=\exp [j \phi(t)], \omega_{0}$ is the carrier, $\phi$ denotes the phase modulation, and $a(t)$ denotes the corresponding bipolar binary amplitude modulation. For the case of a 13-bit Barker biphase code, ${ }^{16}$

$$
\begin{aligned}
& a_{n}(t)=\exp \left[j \phi_{n}(t)\right] \\
& \quad=+1,+1,+1,+1,+1,-1,-1,+1,+1,-1,+1,-1,+1 .
\end{aligned}
$$

For the remainder of this Letter, we consider only the use of the $x=\ln t$ coordinate transformation (because of the Doppler invariance that results) and the 13-bit. Barker coded waveform of Eqs. (4) and (5). No loss of generality results from this choice. Further analysis is merely simpler if a specific example is used.

To demonstrate the factors that affect the choice of the coded waveform used, we apply $t=\exp x$ to Eq. (4), and, with the variable change $x=t$, we find that

$$
f(\exp t)=f_{1}(t)=\cos \left[\omega_{0} \exp t+\phi(\exp t)\right] .
$$

In the conventional space-variant signal-processing approach described above, we would correlate this $f_{1}(t)$ reference signal with a similarly coordinate-transformed received signal $f_{1}{ }^{\prime}(t)$. For the no-distortion case, $f_{1}(t)$ $=f_{1}^{\prime}(t)$, and the resultant autocorrelation is

$$
R(\hat{t})=f(\exp \mathrm{t}) \circledast f(\exp t) .
$$

In Fig. 2(a) we show the cross-sectional scan of an optically produced correlation in Eq. (7) of the nonlinear 13-bit Barker code described by Eq. (6) with bandwidth $=11.4 \mathrm{cycles} / \mathrm{mm}$. Various methods to realize the required coordinate transformation were noted above.
For the data in Fig. 2, a log module was used in the deflection system of a TV monitor. The measured width of the correlation peak (including output display magnification) is $1 \mathrm{~mm}$. To compare this with the performance possible with a linear or normal Barker code of the same bandwidth (BW), we would use a bit length of $2 / 11.4=0.18 \mathrm{~mm}$ and expect an output-correlation peak width or system resolution of $0.36 \mathrm{~mm}$. Experimental data on such a linear Barker code confirmed that such resolution was obtainable on the same optical system. One such typical output is shown in Fig. 2(b). Many other analogous output correlations on various waveforms have been investigated and found to yield similar results. Thus, as was just demonstrated, although the correlation of a nonlinear Barker code as in Eq. (7) results in a good correlation peak, we could achieve nearly three times the performance or resolution (i.e., 1 $\mathrm{mm} / 0.36 \mathrm{~mm}=3$ ) with a normal Barker code of comparable BW.

A stationary phase analysis ${ }^{17}$ of Eq. (7) with typical $\omega_{0}$ and $\phi$ values will show that the nonlinear carrier rather than the phase code determines the BW of $f_{1}(t)$ and all characteristics of $R(\hat{t})$. This is due to the much larger phase change introduced by the nonlinear carrier in Eq. (6). Because of the spread but highly tapered spectrum of Eq. (6), the Doppler resolution $\Delta \nu$ of the system is poor, and better resolution would result if a normal code of the same increased bandwidth were used. Of more concern is the fact that the waveform and system designer now has no tools at his disposal, since changing the phase code $\phi$ in Eq. (6) does not appreciably alter $R(\hat{t})$ in Eq. (7). Similarly, when a range $\tau$ shift is present, the correlation will not be appreciably different, since the major contribution to $R(\hat{t})$ is produced by the coordinate-transformed carrier. This aspect is disastrous when a jammer is present, since it will correlate equally well with $f_{1}(t)$ because both are subjected to the same coordinate transformation. In Fig. 2(b), we show the cross-sectional scan of the correlation of a discrete jammer with the reference waveform in Eq. (6) to demonstrate this. For these and many other reasons, such operation of the system and the use of normal coded waveforms in this type of space-variant processor will produce poor system performance.

The above problems can be alleviated if we transmit a waveform that, when coordinate transformed, results in a normal Barker (or any other) coded signal. For the Mellin-transform coordinate transformation under consideration, the optimum transmitted signal would thus be

$$
f_{t}(t)=\cos \left\{\omega_{0} \ln \left(t+t_{0}\right)+\phi\left[\ln \left(t+t_{0}\right)\right]\right\}
$$

or a $\ln$-scaled version of Eq. (4) with the coordinate transformation

$$
t=\ln \left(t+t_{0}\right)
$$

applied. The $t_{0}$ variable in Eq. (9) is necessary since $\ln$ $0=-\infty$. On reception of $f_{t}(t)$ in Eq. (8) or a Doppleror range-shifted version of it, we would apply the inverse coordinate transformation

$$
t=\exp x-\hat{t}
$$




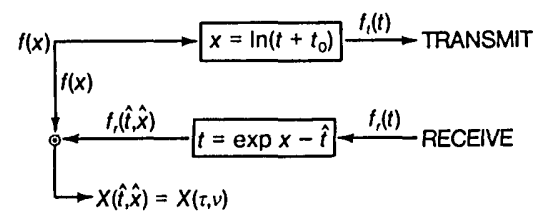

Fig. 3. Block diagram of space-variant optical-ambiguityfunction processor for use with coded waveforms.

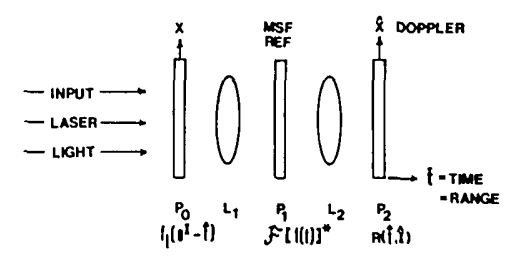

Fig. 4. Schematic diagram of a single-channel space-variant optical processor to calculate the $2-\mathrm{D}$ ambiguity function for coded waveforms.

yielding a new function,

$$
f_{t l}(t)=f_{t}\left(e^{x}-\hat{t}\right)=f_{1}(\hat{t}, x) .
$$

The desirable range and Doppler information for this system are obtained by correlating Eq. (11) with Eq. (4) continuously for all $\hat{t}$ and $\hat{x}$,

$$
R(\hat{t}, \hat{x})=f_{1}(\hat{t}, x) \circledast f(x),
$$

where $\hat{x}$ is the Doppler or correlation variable and $\hat{t}$ is the continuously varying time variable. A block diagram of the final system is shown in Fig. 3.

In this approach, $f(x)$ is coordinate transformed, transmitted, and, on receipt, inverse coordinate transformed; this signal is then correlated with the original $f(x)$ waveform. This approach is far preferable to the correlation of coordinate-transformed versions of the transmitted and received signals, as noted above.

A candidate optical system to realize the functional diagram of Fig. 3 is shown in Fig. 4. One of the realtime and parallel optical space-variant systems noted above is used to realize the transformation from Eq. (8) to Eq. (11). With the transmittance of $P_{0}$ given by Eq. (11) and a matched spatial filter of Eq. (4) recorded at $P_{1}$, the system of Fig. 3 realizes the correlation and ambiguity-function generation described by Eq. (12). In this system, the position in $\hat{x}$ at $P_{2}$ at which the correlation appears will correspond to the target's Doppler, and the time $\hat{t}$ at which the correlation occurs will correspond to the target's range.
The many desirable waveform-design and noiserejection features of this system will be described in later papers. The transmitted waveform in Eq. (12) has many unique features. It uses a nonlinear code, thus decreasing its detectability by a third party. Since the $\mathrm{BW}$ of the original signal is spread and spread nonlinearly, a large noise immunity to jamming and interference results for the same reason that spread-spectrum systems enjoy such noise immunity. The nonlinear coordinate transformation produces a further level of noise and jammer rejection and an increased systemand waveform-design flexibility. Finally, because the carrier in Eq. (8) is nonlinear, its $\omega_{0}$ and pulse-repetition frequency cannot be easily determined.

This work was supported by grant ENG77-20038 from the National Science Foundation.

\section{References}

1. Optical Information Processing-Real-Time Devices and Novel Techniques, Vol. 83 of SPIE Seminar Proceedings (Society of Photo-Optical Inštrumentation Engineers, Bellingham, Washington, 1976).

2. G. M. Robbins and T. S. Huang, Proc. IEEE 60, 862 (1972).

3. A. Sawchuck, J. Opt. Soc. Am. 64, 138 (1974).

4. D. Casasent and D. Psaltis, in Proceedings of the Electro-Optical Systems Design Conference (Milton S. Kiver, Chicago, 1975), p. 38.

5. D. Casasent and D. Psaltis, Opt. Commun. 17, 59 (1976).

6. D. Casasent and D. Psaltis, Proc. IEEE 65, 77 (1977).

7. D. Psaltis and D. Casasent, Appl. Opt. 16, 2288 (1977).

8. D. Casasent and D. Psaltis, Appl. Opt. 15, 2015 (1976).

9. D. Casasent and M. Kraus, Opt. Commun. 19, 212 (1976).

10. O. Bryngdahl, J. Opt. Soc. Am. 64, 1092 (1974).

11. D. Casasent and C. Szczutkowski, Proc. SPIE 83, 91 (1976).

12. D. Casasent and C. Szczutkowski, Opt. Commun. 19, 217 (1976).

13. J. W. Goodman, Proc. IEEE 65, 29 (1977).

14. J. W. Goodman, P. Kellman, and E. W. Hansen, Appl. Opt. 16, 733 (1977)

15. P. Kellman and J. W. Goodman, Appl. Opt. 16, 2609 (1977).

16. C. E. Cook and M. Bernfeld, Radar Signals (Academic, New York, 1967).

17. M. Born and E. Wolf, Principles of Optics (Pergamon, New York, 1965), p. 753. 